\title{
Wolfgang Paulis familiärer Hintergrund
}

Manfred Jacobi*

\section{Summary}

This paper is devoted to the ancestors of the physicist Wolfgang Pauli (1900-1958). The individual members of the family will be introduced and conclusions are drawn on the intellectual situation into which Pauli was born.

\section{Zusammenfassung}

Die vorliegende Arbeit beschäftigt sich mit den Vorfahren des Physikers Wolfgang Pauli (1900-1958). Zunächst werden die einzelnen Mitglieder der Familie vorgestellt, anschliessend sollen daraus Rückschlüsse auf das intellektuelle Klima gezogen werden, in dem der spätere Nobelpreisträger seine Jugend verbrachte.

\section{Einleitung}

Wolfgang Pauli, einer der bedeutendsten Physiker des 20. Jahrhunderts und Entdecker des nach ihm benannten Ausschliessungsprinzips, wurde 1900 in Wien geboren. In dieser Stadt verbrachte er seine gesamte Kindheit und Jugend. Das geistige Klima in der Donaumetropole beeinflusste zweifellos seine intellektuelle Entwicklung. In jenen Jahren formten sich die Fundamente seiner Persönlichkeit, die ihn zu einer unverwechselbaren Erscheinung innerhalb der Scientific Community werden liessen. In der vorliegenden

* Der Autor dankt Prof. Dr. Christoph Meinel und Dr. Karl von Meyenn für wertvolle Anregungen bei der Abfassung dieser Arbeit.

Abgekürzt zitiert wird: $P W B=$ Wolfgang Pauli, Wissenschaftlicher Briefwechsel mit Bohr, Einstein, Heisenberg u.a. (Berlin/Heidelberg/New York 1979ff.).

Dr. Manfred Jacobi, Rue Archimède 87/4, B-1000 Bruxelles, Belgium

(Manfred.Jacobi@web.de). 
Arbeit soll versucht werden, durch die Untersuchung seines familiären Umfeldes Erkenntnisse zu gewinnen über das geistige Milieu, in dem Wolfgang Pauli die Zeit seiner Adoleszenz durchlebte.

Der väterliche und der mütterliche Teil der Familie haben sehr unterschiedlich auf die Selbstformung des späteren Physikers gewirkt. Die Mutter, die einem künstlerischen Haus entstammte und journalistisch tätig war, hat wohl vor allem die musische Seite in Wolfgang Paulis Wesen geprägt. Ähnliches gilt auch für deren Eltern, Bertha und Friedrich Schütz, die dem Theater bzw. der Literatur verbunden waren. Der Vater, selbst naturwissenschaftlich tätig, war dagegen sehr um die gründliche mathematisch-physikalische Ausbildung seines Sohnes bemüht.

Im folgenden sollen die einzelnen Zweige der Familie Pauli dargestellt werden. Dabei interessiert einerseits der Lebenslauf der jeweiligen Personen, andererseits soll besonderes Augenmerk auf schriftliche Zeugnisse gelegt werden, die sie hinterlassen haben und die einen Einblick in ihre Geisteswelt erlauben. Natürlich kommen auch andere Quellen zu Wort, in denen die Vertreter dieser Personengruppe von dritter Seite charakterisiert werden. Ein weiterer Schwerpunkt der Arbeit besteht darin, Querverbindungen zwischen den Erkenntnissen über die einzelnen Familienmitglieder und den sehr charakteristischen Persönlichkeitsmerkmalen des Physikers Wolfgang Pauli zu beleuchten und daraus - mit der gebotenen Vorsicht Rückschlüsse auf eventuelle Einflussfaktoren zu ziehen.

\section{Die Familie der Mutter}

\section{Der Grossvater Friedrich Schütz}

Wolfgang Paulis Grossvater mütterlicherseits, Friedrich Schütz (1845-1908), wurde am 24. April 1845 in Prag geboren. Seine schulische Ausbildung genoss er bei den Piaristen, einem katholischen Orden, der sich insbesondere dem Schulunterricht und der Jugenderziehung widmete ${ }^{1}$. Nach dem Willen seiner Eltern sollte er Geistlicher werden, jedoch neigte er eher dem Theater und der Literatur zu. Seinen ersten «dramatischen Scherz» verfasste er als Sechzehnjähriger. Es folgten weitere Theaterstücke, wobei das 1869 erschienene Lustspiel Gegenseitig ihm auch öffentliche Anerkennung brachte. Bestimmt hat ihn sein erster Erfolg dazu animiert, weitere Stücke für die Bühne zu schreiben. Überhaupt scheint ihn die dramatische Form

1 R. Hemmerle, in: Mitteilungen des Sudetendeutschen Archivs (München) 119 (1995) 33. 
zeitlebens nicht losgelassen zu haben. So spricht er einmal selbst von dem «auf die Phantasie mächtig wirkenden Theaterapparate und [...] dem Reize der sinnlichen Bühnenatmosphäre»². Das Theater ist für ihn keineswegs nur Selbstzweck. Vielmehr betrachtet er es als ein Abbild der gesellschaftlichen Auseinandersetzungen im Fin de Siècle und als ein Mittel zur Ausbreitung fortschrittlich-liberaler Ideen.

Friedrich Schütz wuchs in einer Epoche des gesellschaftlichen und politischen Umbruchs auf. Der Kampf des Neuen gegen das Alte, der für seine Generation kennzeichnend ist, offenbart sich nahezu permanent in seinem literarischen Schaffen. Es ist sehr charakteristisch für den Gesellschaftskritiker Schütz, dass er in seinen Erörterungen auch Querbezüge zu politischen Ereignissen herstellt. Dem fortschrittsgläubigen Freidenker ist jede Form von Rückwärtsgewandtheit verdächtig. Schonungslos spürt er überall das Wirken reaktionärer Kräfte auf, auch in seinem eigenen Tätigkeitsfeld, dem literarischen Schaffen. Im Wien des ausgehenden 19. Jahrhunderts wird er in dieser Hinsicht sehr leicht fündig ${ }^{3}$. Auf allen Gebieten des Lebens versucht er «den Zusammenhang der grossen Weltereignisse mit den Erscheinungen der Literatur nachzuweisen» ${ }^{4}$. Dabei kommt dem damals sehr mächtigen Einfluss des Katholizismus auf das österreichische Geistesleben eine besondere Bedeutung zu. An seiner antiklerikalen Haltung in dieser Frage lässt er jedenfalls keinen Zweifel aufkommen.

Noch in Prag wurde Schütz Korrespondent der Neuen Freien Presse, die, nach der Revolution von 1848 gegründet, sich rasch als modernes, weltoffenes Blatt etablierte, das während der Ringstrassenepoche seine Hochblüte erlebte. Seit 1873 war er als Redakteur dieser bedeutendsten liberalen Zeitung der k.u.k. Monarchie in Wien tätig. Mehr als dreissig Jahre lang sollte er ihr verbunden bleiben. Die Wahl der Neuen Freien Presse war keineswegs zufällig, hatte Schütz doch schon in seiner Prager Zeit den Aufstieg des österreichischen Liberalismus miterlebt und enge Kontakte zu liberalen Politikern geknüpft ${ }^{5}$. Trotz seiner geradezu fanatischen Freigeisterei und seiner tiefen Verbundenheit mit dem österreichischen Liberalismus konnte er sich nicht dazu entschliessen, eine politische Karriere einzuschlagen. Im Gegenteil: das Aufkommen starker reaktionärer Kräfte bewirkte eine Zurück-

2 F. Schütz, Wiener Theater-Eindrücke.Vortrag, gehalten im Verein der Literaturfreunde in Wien (Wien 1896) 4.

3 Einen Einblick in die sozialen, kulturellen und politischen Verhältnisse im Wien der Jahrhundertwende vermittelt Carl E. Schorske in seinem Buch Wien - Geist und Gesellschaft im Fin de Siècle (München 1994).

4 Schütz, Theater-Eindrücke (oben Anm. 2) 21.

5 F. Schütz, Werden und Wirken des Bürgerministeriums - Mitteilungen aus unbenutzten Quellen und persönliche Erinnerungen (Leipzig 1909) VI. 
drängung des liberalen Einflusses in Österreich, was den scharfzüngigen Literaten dazu veranlasst haben mag, der Politik den Rücken zu kehren. So konzentrierte er sich verstärkt auf seine eigentliche Berufung, das Schreiben. In seiner schriftstellerischen Tätigkeit spielte das politische Geschehen, zumindest vordergründig betrachtet, nur eine bescheidene Rolle. Einer der Schwerpunkte seiner redaktionellen Tätigkeit war die Theaterkritik. Allerdings verbarg sich hinter dieser Maske des Kritikers doch stets nur der unerbittliche Analytiker der politischen Wetterlage. Neben seinem Engagement gegen den Klerikalismus war der langjährige und nicht immer von Erfolg gekrönte Kampf seiner Zeitgenossen gegen die allmächtige Zensur eines seiner bevorzugten Themen.

Im Jahre 1894 wurde er als Berichterstatter seiner Zeitung zur Krönung von Zar Nikolaus II. nach Russland entsandt. Bei dieser Gelegenheit besuchte der österreichische Gast auch Leo Tolstoi. Die Begegnung mit dem grossen russischen Schriftsteller scheint für den Wiener Zeitungskorrespondenten der eigentliche Höhepunkt seiner Reise gewesen zu sein. Noch auf der Rückfahrt von Tolstois Landgut erschien ihm dessen Haus «wie ein Wahrzeichen, dass kühne Geister über die Macht der Finsternis siegen werden ${ }^{6}$.

In seinem letzten und umfassendsten literarischen Werk mit dem Titel Werden und Wirken des Bürgerministeriums beschäftigte sich Friedrich Schütz mit jener kurzen historischen Episode, in der der Liberalismus in Österreich nicht nur wirtschaftlich, sondern auch politisch den Ton angab? Der Grundtenor dieses Buches spiegelt nicht nur seine liberale Gesinnung wieder. Vielmehr spielen dabei auch persönliche Erinnerungen sowie seine engen Kontakte zu einigen Mitgliedern des Bürgerministeriums eine wichtige Rolle. Die erste und einzige liberale Regierung in der Geschichte Österreichs war bis Anfang April 1870 im Amt. Schütz hebt nicht nur die besondere politische Bedeutung dieser kurzen Epoche hervor, er unterstreicht auch die Art und Weise, in der die liberale Geistesströmung alle Bereiche des Lebens erfasste.

Die schriftlichen Zeugnisse, die uns Friedrich Schütz hinterlassen hat, zeigen uns ihren Verfasser als einen Mann mit klaren gesellschaftspolitischen Prinzipien. Seine Liebe galt, von frühester Jugend an, dem geschriebenen Wort, das er meisterhaft einzusetzen verstand. Als überzeugter Freidenker war ihm die schonungslose Kritik aller von ihm als einengend empfundenen Tendenzen ein zentrales Anliegen. In einem Nachruf auf seine Person heisst

6 F. Schütz, Das heutige Russland (Leipzig 1897) 93.

7 Das sogenannte Bürgerministerium wurde nach dem Abschluss des Ausgleichs zwischen Österreich und Ungarn im Dezember 1867 gebildet. Es verkörperte die Regierung der österreichischen Reichshälfte und stützte sich im wesentlichen auf Vertreter der deutsch-österreichischen Liberalen. 
es: «Er war [...] ein Kritiker mit Hieben, die bis zum Sattelknopfe trafen. Und noch dazu ein durch und durch gescheiter Mensch, dessen lichter Verstand jedem unentbehrlich wurde, dem er in Freundschaft zugetan war.» Darüber hinaus ist die Rede von seinem «wunderbaren Auffassungsvermögen, seiner Fähigkeit, anzuregen und anzufeuern und seiner beneidenswerten Gabe, sich Freunde zu machen ${ }^{8}$. Die Bedeutung, die der Journalist und Schriftsteller Friedrich Schütz innerhalb des Wiener Geisteslebens besass, lässt sich unter anderem daran ermessen, dass seine publizistischen Äusserungen des öfteren zur Zielscheibe ironischer Attacken in der von Karl Kraus herausgegebenen Zeitschrift Die Fackel wurden. Friedrich Schütz starb am 22. Dezember 1908 in Wien.

\section{Die Grossmutter Bertha Schütz.}

Wolfgang Paulis Wiener Grossmutter Bertha Schütz (1847-1916), geb. Dillner von Dillnersdorf, kam am 10. November 1847 als Tochter des k.k. Polizei-Oberkommissärs Otto Dillner von Dillnersdorf in Wien zur Welt ${ }^{9}$. Schon frühzeitig zeigte sich ihr musikalisches und gesangliches Talent. So sang sie als 13jährige in Konzerten, erhielt eine gründliche stimmliche Ausbildung und kam als Elevin an die k.k. Hofoper, wo sie von Juli 1865 bis Mai 1866 blieb. Im Anschluss daran wechselte sie an die Kölner Oper, der sie drei Jahre lang angehörte. An dieser Bühne feierte sie ihre ersten Triumphe als Sopranistin. Anschliessend erhielt sie ein Engagement am Deutschen Landestheater in Prag. Dort debütierte sie als Ännchen im C. M. von Webers Freischütz und als Martha in F. v. Flotows gleichnamiger Oper. Ende Dezember 1872 nahm sie Abschied vom Prager Bühnenleben, um an die Wiener Hofoper zu wechseln. Zur gleichen Zeit wurde ihr späterer Ehemann Friedrich Schütz von Prag nach Wien versetzt, um von da an in der dortigen Redaktion der Neuen Freien Presse zu arbeiten. 1875 vermählte sich die gefeierte Sopranistin mit dem freidenkerischen Schriftsteller. Die k.k. Hofoper, an der sie in G. Meyerbeers Les Huguenots debütierte, war zweifellos das bedeutendste österreichische Opernhaus. Ihm gehörte sie bis zur Beendigung ihrer Gesangskarriere im Jahre 1885 an. Während dieser Zeit verkörperte sie zahlreiche Figuren des lyrischen und dramatischen Sopran- und Mezzosopranfachs. Höhepunkte ihres Bühnenlebens waren ihre Auftritte

8 Schütz, Bürgerministerium (oben Anm. 5) VI.

9 Ludwig Eisenberg, Grosses Biographisches Lexikon der Deutschen Bühne (Leipzig 1903) und Walther Killy (Hrsg.), Deutsche Biographische Enzyklopädie 2 (München u.a. 1995). 
in Mozarts Don Giovanni und in der Zauberflöte. Darüber hinaus trat sie auch als Wagner-Sängerin hervor. Neben ihrer Tätigkeit für Wiens führendes Musiktheater gab sie auch zahlreiche Gastspiele ausserhalb des österreichischen Musiklebens, vor allem in Deutschland. Überall wurden der Wohllaut ihrer Stimme, die Kraft und Wärme ihres Tones sowie ihr musikalischer Sinn und ihre physische Ausdauer gelobt. Schliesslich fand auch ihre schauspielerische Begabung einhellige Anerkennung. Ihr früher Abschied vom Bühnenleben wurde auf ihre «hochgradige Nervosität» ${ }^{10}$ zurückgeführt. Nach ihrem Abschied wurde sie zum Ehrenmitglied der Hofoper ernannt. Bertha Schütz starb am 27. Juli 1916 in Wien.

\section{Die Mutter Bertha Pauli}

Aus der Ehe von Friedrich und Bertha Schütz gingen zwei Töchter hervor. Die Mutter des Physikers und Nobelpreisträgers, Bertha Kamilla Pauli, geb. Schütz (1878-1927), kam am 29. November 1878 in Wien zur Welt. Sie wuchs in einer literarisch-künstlerischen Familie auf und wurde schon frühzeitig mit freisinnigem Gedankengut vertraut. Sie war ebenso wie ihr Vater im Bereich des Journalismus tätig. Wie dieser arbeitete sie für die Neue Freie Presse und verfügte über ein breites Repertoire sprachlicher Ausdrucksmittel. Während sich in ihren Artikeln vielfach der Einfluss der väterlichen Gedankenwelt nachweisen lässt, entwickelte sie dennoch in hohem Masse ein eigenes Profil, das sich in markanter Weise von dem ihres Vaters unterschied. Wie dieser stand sie den Aktivitäten der katholischen Kirche äusserst kritisch gegenüber. 1911 trat sie zur evangelischen Glaubensgemeinschaft über. Die genauen Beweggründe für diesen Schritt sind unbekannt.

Für die Neue Freie Presse verfasste sie eine Reihe von Essays über historische Themen sowie theaterkritische Aufsätze. Eines ihrer bevorzugten Themen war der Kampf für die Rechte der Frauen. So beschäftigte sie sich in ihren historischen Essays unter anderem mit bedeutenden Frauengestalten während der Zeit der Französischen Revolution, mit dem Prozess gegen Jeanne d'Arc und mit der Dichterin George Sand. Daneben galt ihr Engagement dem Pazifismus, der sozialen Gerechtigkeit und dem Kampf gegen die Reaktion, den schon ihr Vater wortgewaltig geführt hatte. Diese Ziele schienen ihr am besten von den österreichischen Sozialisten verfochten zu werden. Vor diesem Hintergrund verwundert es nicht, dass sie auch für die Arbeiter-Zeitung, das Zentralorgan der sozialdemokratischen Partei,

10 Eisenberg, a.O. (oben Anm. 9) 201. 
schrieb. Im Wahlkampf des Jahres 1919 rief sie die österreichischen Frauen dazu auf, sozialdemokratisch zu wählen, um nicht weiterhin «das gefügige Werkzeug der Reaktion»" zu bleiben.

Die Fortschrittsgläubigkeit, die schon ihr Vater in bezug auf den Liberalismus gezeigt hatte, findet sich in entsprechender Weise bei Bertha Pauli, wobei sie nunmehr den Sozialismus für die Triebfeder des Fortschritts hielt. Auch was ihr pazifistisches Engagement betrifft, vertrat sie die Ansicht, dass eine friedliche Zukunft «nur der unwiderstehliche Strom proletarischer Massenbewegung erreichen und verwirklichen kann» ${ }^{12}$. Mit überzeugender Anteilnahme berichtete sie von internationalen Friedensaktivitäten, die nach dem Ende des Ersten Weltkriegs den Grundstein für eine friedliche Zukunft der Welt legen wollten. Die grosse soziale Not der 20er Jahre wirkte sich besonders verhängnisvoll auf viele Kinder aus. Dies führte zu der Forderung, man möge die Jugendfürsorge stärken und so das Schicksal des jungen Lebens in geordnete Bahnen lenken. Auch diese Bevölkerungsgruppe fand in Bertha Pauli eine entschiedene Fürsprecherin.

In ihren theaterkritischen Artikeln zeigt sie sich als eine interessierte und sachkundige Journalistin, die - ebenso wie ihr Vater - vom Theater wesentlich mehr erwartete als nur Erbauung und Zerstreuung. Für sie war die Bühne ein Werkzeug für den gesellschaftlichen Fortschritt. Einer ihrer Interessensschwerpunkte galt dem zeitgenössischen Drama, das die aktuellen gesellschaftlichen Entwicklungen und die damit verbundenen persönlichen Schicksale thematisierte. Offene Kritik übte sie, wenn die Wiener Theater ihre Türen vor diesen modernen Stücken verschlossen.

Bertha Pauli schied am 15. November 1927 freiwillig aus dem Leben. Einem Nachruf auf ihre Person entnehmen wir, dass sie an pathologischen Angstzuständen litt, die anfallsartig auftraten ${ }^{13}$. In der Nacht vom 12. auf den 13. November hatte sie eine Überdosis des Beruhigungsmittels Veronal zu sich genommen, an der sie zwei Tage später starb. Ihre Kollegen von der Arbeiter-Zeitung lobten sie als «eine tapfere Frau» und als «entschlossene Sozialistin». Ein weiteres Markenzeichen der streitbaren Journalistin war ihre direkte und unverblümte Kritik an bestehenden Missständen der jungen Republik. Sie nahm kein Blatt vor den Mund, wenn es galt, das als richtig Erkannte einer breiteren Öffentlichkeit mitzuteilen, auch wenn dies vielen Zeitgenossen zuwiderlaufen musste. In einem anderen Nachruf ist von ihrer «peinlichen Selbstkritik, verbunden mit einem stark ausgeprägten Minder-

11 B. Pauli, in: Arbeiter-Zeitung vom 2. Febr. 1919.

12 B. Pauli, in: Arbeiter-Zeitung vom 23. Mai 1921.

13 Arbeiter-Zeitung vom 18. Nov. 1927. 
wertigkeitsgefühl» die Rede, was sie - so wird gemutmasst - zu ihrem «verhängnisvollen Schritt» getrieben haben soll ${ }^{14}$. In diesem Zusammenhang muss allerdings darauf hingewiesen werden, dass die Ehe der Paulis zu dieser Zeit in eine schwierige Lage geraten war. Wolfgang Pauli sen. hatte kurz zuvor die junge Bildhauerin Maria Rottler kennengelernt ${ }^{15}$, die er bereits ein halbes Jahr nach dem Tod seiner ersten Frau heiratete.

\section{Die Vorfahren des Vaters}

Der väterliche Zweig der Familie hatte seine Wurzeln im Prager Judentum. Wolfgang Paulis Urgrossvater Wolf Pascheles (1814-1857) stammte aus ärmlichen Verhältnissen. Er betätigte sich bereits in jungen Jahren als Verleger von jüdischen Gebetbüchern und vertrieb seine Produkte als fliegender Händler. 1837 eröffnete er schliesslich eine Buchhandlung in Prag ${ }^{16}$. Die Spezialisierung auf die Herausgabe von Judaica erwies sich als eine zukunftsweisende Entscheidung. Ein besonderer Erfolg war die 1846 erstmals erschienene Sammlung mit dem Titel Sippurim, die unter anderem jüdische Volkssagen sowie Biographien berühmter Juden enthielt. Darüber hinaus umfasste die publizistische Tätigkeit von Wolf Pascheles unter anderem auch den beliebten Illustrirten Israelitischen Volks-Kalender und den Pentateuch mit einer deutschsprachigen Übersetzung. Seine Söhne Samuel und Jakob W. Pascheles (1839-1897) waren ebenfalls im Buchwesen tätig. Jakob übernahm nach dem Tod seines Vaters die Leitung des Verlages, dessen Geschäft weiterhin auf den Vertrieb von jüdischer Literatur beschränkt blieb. Daneben führten sie auch die Buchhandlung ihres Vaters weiter. Jakob Pascheles heiratete Helene Utitz, die Tochter einer alteingesessenen jüdischen Familie Prags, deren böhmische Wurzeln sich bis an den Anfang des 17. Jahrhunderts zurückverfolgen lassen ${ }^{17}$. Aus dieser Ehe gingen nicht weniger als fünf Kinder hervor, wobei unser Interesse dem 1869 zur Welt gekommenen Wolf Pascheles gilt.

14 Die Frau, wahrscheinlich Dezember 1927. Eine Kopie dieses Nachrufs verdanke ich Frau Dr. Elisabeth Lebensaft vom Institut Österreichisches Biographisches Lexikon.

15 Die Stunde, 4. Okt. 1935.

16 The Jewish Encyclopedia 9 (New York, o.J.) $546 \mathrm{f}$.

17 Gerda Hoffer, Ererbt von meinen Vätern. 400 Jahre europäisches Judentum im Spiegel einer Familiengeschichte (Köln 1990) 86. 


\section{Der Vater Wolfgang Joseph Pauli, geb. Wolf Pascheles}

Wolf Pascheles, der Vater von Wolfgang Pauli, wurde am 11. September 1869 in Prag geboren. Er besuchte seit dem Schuljahr 1879/80 das Altstädter Gymnasium in Prag. Die Entscheidung, diese deutschsprachige Schule zu besuchen, mag zum Teil damit im Zusammenhang gestanden haben, dass sich das Schulgebäude in unmittelbarer Nähe zum Wohnsitz der Pascheles am Altstädter Ring befand ${ }^{18}$. Grundsätzlich lässt sich in jenen Jahren eine grössere Affinität der jüdischen Bevölkerung zu deutschen als zu tschechischen Schulen feststellen ${ }^{19}$. Am Altstädter Gymnasium legte Wolf nach acht Schuljahren seine Reifeprüfung im Juli 1887 ab. Noch im gleichen Jahr immatrikulierte er sich an der deutschen Universität in Prag, um das Studium der Medizin aufzunehmen.

Wolf Pascheles hatte bereits während seiner Gymnasialzeit Ludwig Mach, einen Sohn des grossen österreichischen Physikers und Philosophen Ernst Mach, kennengelernt. Beide nahmen auch gleichzeitig das Studium der Medizin an der deutschen Universität in Prag auf. Es erscheint plausibel anzunehmen, dass Wolf die eine oder andere Lehrveranstaltung seines späteren Mentors Ernst Mach, der zu dieser Zeit ebenfalls an der CarlFerdinands-Universität lehrte, belegte ${ }^{20}$. Noch vor seiner Promotion zum «Doktor der gesamten Heilkunde» am 24. April 1893 hatte Wolf Pascheles seine ersten wissenschaftlichen Arbeiten veröffentlicht, von denen wenigstens eine im physikalischen Institut der Prager Universität unter Ernst Mach entstanden war $^{21}$. Die lebenslange Verbundenheit zwischen den beiden Wissenschaftlern dürfte hier ihren Ausgang genommen haben.

Kurze Zeit nach seiner Promotion übersiedelte Wolf Pascheles im Juni 1893 nach Wien. Dort blieb er fünf Jahre lang als Assistentsarzt am k.k. Rudolfsspital. Diese Zeit nutzte er neben seinen ärztlichen Aufgaben auch zur wissenschaftlichen Forschung. Viele seiner Arbeiten behandeln physikalische Probleme, angewendet auf tierische oder menschliche Organismen. So verwundert es nicht, dass ihm im Rahmen seines Habilitationsverfahrens eine «vortreffliche mathematisch-physikalische Schulung» und darüber hinaus die Beherrschung der «modernen physikalischen Methoden» bescheinigt wurde ${ }^{22}$. Im Januar 1899 habilitierte er sich für innere Medizin. Damit war das Tor zu seiner weiteren akademischen Laufbahn geöffnet.

18 F. Smutny, «Ernst Mach and Wolfgang Pauli’s Ancestors in Prague», Gesnerus 46 (1989) 183-194.

19 R. M. Wlaschek, Juden in Böhmen (München 1990) 25.

20 Smutny, a.O. (oben Anm. 18).

21 Akten des Wiener Universitätsarchivs über W. Pauli sen.

22 Akten des Wiener Universitätsarchivs über W. Pauli sen. 
In die Zeit des Habilitationsverfahrens fiel auch eine grundlegende Entscheidung für das weitere Leben des Wolf Pascheles. Nach dem Tod seines Vaters Jakob fasste er den Entschluss, zum Katholizismus überzutreten. Ende Juli 1898 änderte er seinen Familiennamen von Pascheles in Pauli. Den endgültigen Abschied von seinen jüdischen Wurzeln besiegelte er im März 1899 mit dem Austritt aus dem Judentum. Die Gründe für diesen Schritt mögen vielfältig gewesen sein: Zum einen war ein Übertritt zum Katholizismus in dieser Epoche der Habsburgermonarchie seiner Karriere durchaus förderlich $^{23}$. Zum anderen dürfte er zu diesem Zeitpunkt bereits seine spätere Ehefrau Bertha Kamilla Schütz gekannt haben, die ebenfalls katholisch war. Die kirchliche Trauung fand nur wenige Wochen nach seiner Loslösung vom jüdischen Glauben statt, und zwar am 2. Mai 1899. Angesichts der tiefen Verwurzelung seiner Prager Vorfahren in der jüdischen Tradition mag der Umstand, dass sein Vater Jakob Pascheles schon 1897 gestorben war, dem jungen und zielstrebigen Mediziner die Entscheidung erleichtert haben.

Es folgte eine erfolgreiche und überaus produktive akademische Laufbahn. 1903 übernahm Wolfgang Pauli sen. die Leitung der physikalisch-chemischen Abteilung der Biologischen Versuchsanstalt im Wiener Prater. Zehn Jahre später erhielt er eine ausserordentliche Professur an der Universität Wien und wurde Vorstand des Institutes für physikalisch-chemische Biologie. 1920 erhielt er den Titel eines ordentlichen Professors. 1922 schliesslich wurde er zum Vorstand des Instituts für medizinische Kolloidchemie ernannt, das gerade erst neu eingerichtet worden war. Seine wissenschaftlichen Leistungen wurden mehrfach honoriert; so erhielt er 1917 den Haitinger-Preis der Kaiserlichen Akademie der Wissenschaften und sechs Jahre später den Leonard-Preis der Kolloid-Gesellschaft. Schliesslich wurde er 1934 korrespondierendes Mitglied der Österreichischen Akademie der Wissenschaften. Nach dem Anschluss Österreichs im Jahre 1938 musste er seine Heimat aus rassischen Gründen verlassen. Er ging in die Schweiz, wo auch sein Sohn lebte, und liess sich in Zürich nieder. Auch nach seiner Emigration ging er weiter seinen Forschungsarbeiten nach.

Wolfgang Pauli sen. gehörte zu den Pionieren auf dem Gebiet der Biophysik und der Kolloidchemie. Schwerpunkte seiner wissenschaftlichen Arbeit waren die Chemie der Proteine und die Kolloidchemie anorganischer Sole. Er strebte danach, den Zusammenhang zwischen dem physikalischchemischen Verhalten der Eiweisse und ihrer Konstitution aufzuklären. Neben einer Vielzahl wissenschaftlicher Artikel veröffentlichte er auch Bücher über sein Fachgebiet. Die beiden wichtigsten sind die Kolloidchemie der 
Eiweisskörper (1920) und die Elektrochemie der Kolloide (1929, zusammen mit E. Valko). Gemeinsam mit einem anderen Kollegen, dem Nobelpreisträger für Medizin Karl Landsteiner, entwickelte Pauli einen elektrischen Überführungsapparat. Darüber hinaus erfand er Apparaturen zur Elektrodialyse und zur Elektrodekantation, die es ermöglichten, Eiweisskörper in höchster Reinheit herzustellen. Wolfgang Joseph Pauli starb am 4. November 1955 in Zürich.

\section{Querverbindungen zu Wolfgang Pauli}

Im folgenden soll nach den Spuren der Einflüsse auf den Physiker durch sein familiäres Umfeld gesucht werden. Paulis eigene Angaben über seinen familiären Hintergrund sind relativ spärlich, so dass viele Schlussfolgerungen auf sorgfältigen Vergleichen zwischen dem, was über seine Person bekannt ist, einerseits und den oben dargelegten Erkenntnissen über die ihm nahestehenden Menschen andererseits beruhen.

Zunächst möchten wir unser Augenmerk auf eine charakteristische Ähnlichkeit der Persönlichkeitsstruktur zwischen Grossvater und Enkel legen. Dabei soll an die erwähnte Kritikfähigkeit von Friedrich Schütz erinnert werden. Augenscheinlich ist vor diesem Hintergrund der Vergleich des Schriftstellers mit dem Physiker Wolfgang Pauli, dessen offene und harte, aber stets sachliche Kritik bei seinen Kollegen weithin bekannt und geschätzt war. Bezeichnungen wie «Meister der Kritik» (Heisenberg) und «Geissel Gottes» (Ehrenfest) geben uns eine Vorstellung von der Wirkung, die Paulis Kritikfähigkeit in Physikerkreisen hinterliess. Gleichwohl war der Entdecker des Ausschliessungsprinzips ein gern gesehener Gast bei Diskussionen über physikalische Probleme, und vielen Kollegen, die seine unnachsichtige Kritik zu spüren bekamen, war er in langjähriger Freundschaft verbunden. Ebenso muss an dieser Stelle auf die Offenheit und Direktheit verwiesen werden, mit der die Mutter des Physikers, Bertha Pauli, ihre journalistischen Fähigkeiten einsetzte, um aus ihrer Sicht dem Fortschritt der Menschheit zu dienen.

Für die Biographie Paulis ist im Zusammenhang mit dem Nachlass des

Schriftstellers Friedrich Schütz besonders ein Landhaus in Steinbach am Attersee von Interesse. Es kann als sicher angenommen werden, dass Wolfgang dieses Haus, das nach dem Tod seines Grossvaters zum Teil an ihn ging, das eine oder andere Mal besucht hat. Möglicherweise war es auch Zwischenstation auf den Reisen zu seinem Patenonkel Ernst Mach, den er zusammen mit seinem Vater gelegentlich in Vaterstetten bei München 
besuchte. Wolfgang Pauli besass eine ausgeprägte Neigung, sich mit psychologischen Fragestellungen zu beschäftigen, was nicht zuletzt durch seinen umfangreichen Briefwechsel mit dem Schweizer Psychoanalytiker C. G. Jung dokumentiert ist. Pauli beschäftigte sich häufig mit der Deutung seiner eigenen Träume. In einigen dieser Träume spielt das erwähnte Haus des Grossvaters eine gewisse Rolle, so etwa, wenn vom «Landhaus meines Grossvaters» ${ }^{24}$ und von der «Landschaft am Attersee» ${ }^{25}$ die Rede ist. Auf das Grossstadtkind Wolfgang scheint die idyllische Gegend des Salzkammergutes einen bleibenden Eindruck hinterlassen zu haben.

Auch die Grossmutter spielt gelegentlich in Wolfgang Paulis Träumen eine Rolle, und zwar in der Figur einer Klavierspielerin. Gegen Jahresende 1953 verfasste der Physiker eine umfangreiche Schrift mit dem Titel Die Klavierstunde. Eine aktive Phantasie über das Unbewusste ${ }^{26}$. Darin versetzt er sich in Gedanken um 40 Jahre zurück in seine Vaterstadt Wien. Eine «sehr vornehme Dame» mit «dunklen Haaren» gibt dem Dreizehnjährigen eine Klavierstunde. Es wird berichtet, dass Paulis Grossmutter ihrem Enkel des öfteren auf dem Klavier vorgespielt hat ${ }^{27}$. Es liegt deshalb nahe, die erwähnte «Dame» mit der Grossmutter des Physikers zu assoziieren. Noch ein weiterer Punkt scheint diese Annahme zu stützen. In der aktiven Phantasie heisst es an einer Stelle: «Die Dame [...] sprang vom Stuhl und lief im Zimmer einige Male ganz aufgeregt auf und ab. Dann setzte sie sich wieder neben mich $[\ldots] »^{28}$. Dieses Verhalten der klavierspielenden «Dame» erinnert uns an die «hochgradige Nervosität» von Bertha Schütz-Dillner.

In einem Traum vom August 1953 kommen mehrere Gestalten vor, die möglicherweise Ähnlichkeiten mit Personen aus seinem familiären Umfeld besitzen. Pauli beschreibt die entsprechende Szene wie folgt:

Ein Mann stellt in chemischen Retorten aus radioaktivem Uran eine andere gelbe Substanz her, die so aussieht wie Brom. Dann verschwindet der Mann. Zwei anwesende Frauen (davon eine ein mütterlicher Typus) fangen an, aus Angst zu schreien. Sie sagen, der verschwundene Mann sei ein längst Verstorbener, ein Totengeist. Die jüngere fügt noch hinzu, sie habe deshalb so Angst, weil er die Streifen hervorbringe.

Ich widerspreche den beiden, sage es sei gar kein Toter gewesen und ich sähe auch gar keine Streifen. Die Frauen beruhigen sich etwas ${ }^{29}$.

24 Pauli an von Franz, 4. Sept. 1953 (PWB IV/2, 259).

25 Pauli an von Franz, 6. Nov. 1953 (PWB IV/2,343).

$26 P W B$ IV $/ 2,330-340$.

27 C. P. Enz, «Rationales und Irrationales im Leben Wolfgang Paulis», in: H. Atmanspacher et al. (Hg.), Der Pauli-Jung-Dialog und seine Bedeutung für die moderne Wissenschaft (Berlin/Heidelberg 1995) 22.

$28 \mathrm{PWB} \mathrm{IV} / 2,331$.

$29 P W B \mathrm{IV} / 2,251$. 
Das ängstliche Verhalten der Frauen weckt Assoziationen mit den oben erwähnten pathologischen Angstzuständen, unter denen seine Mutter gelitten haben soll. Aber auch eine Verbindung mit der Nervosität der Grossmutter erscheint nicht völlig abwegig. Der Mann mit den chemischen Retorten erinnert in diesem Zusammenhang an Wolfgang Paulis Vater, der auf dem Gebiet der Kolliodchemie tätig war.

Wolfgang Pauli hat einen umfangreichen Briefwechsel hinterlassen. Seine sprachliche Ausdrucksfähigkeit meistert auch komplizierte Sachverhalte wie etwa die Diskussion quantenphysikalischer Probleme mit beeindruckender Eleganz und Klarheit. Auch seine wissenschaftlichen Arbeiten zeigen ihn als einen Autor, der über ein vielfältiges sprachliches Repertoire verfügt, das es ihm erlaubt, seine Ideen mit grosser Übersicht und Systematik darzustellen. Der sichere und gekonnte Umgang mit der deutschen Sprache besass in der Familie Pauli, wie wir gesehen haben, durchaus Tradition und gehörte gewissermassen zum Broterwerb. Vor diesem Hintergrund überrascht es nicht, dass die Fähigkeit zum professionellen schriftlichen Ausdruck bereits den jungen Physiker auszeichnete. Besonders wortgewaltig wurden seine Formulierungen, wenn es darum ging, die wissenschaftliche Welt vor folgenschweren Irrtümern zu bewahren, wie etwa sein Briefwechsel mit Bohr und anderen Kollegen während der Debatte um die Bohr-Kramers-SlaterTheorie eindrucksvoll belegt. Auch Wolfgangs Grossvater sowie seine Mutter erreichten eine überaus prägnante Ausdrucksfähigkeit, wenn es darum ging, Fehlentwicklungen zu brandmarken.

Trotz mancher erkennbarer Spuren, die sowohl die Grosseltern als auch die Mutter in Paulis Persönlichkeit hinterlassen haben, gibt es auch signifikante Unterschiede. Am bemerkenswertesten ist wohl der Umstand, dass der Physiker sich - wohl schon seit seinen jungen Jahren - weitgehend von Zeitungen ferngehalten hat. Mehrfach finden sich in seinem Briefwechsel und in zeitgenössischen Berichten eindeutige Hinweise darauf, dass er keine Zeitungen gelesen hat. Bei einer Gelegenheit bezeichnete er das Zeitunglesen sogar als «Laster» ${ }^{30}$ und wies darauf hin, dass diese Druckerzeugnisse eine «Manifestation des kollektiven Unbewussten» seien. An anderer Stelle stellte er dazu erläuternd fest, «dass Sie, sobald Sie Zeitung lesen, Ihren Namen (d.h. Ihre Individualität) verlieren und gänzlich kollektiv werden $»^{31}$. Dieses eigentümliche Verhältnis zur Presse ist um so erstaunlicher, als die beiden erwähnten Familienmitglieder fast ihr gesamtes berufliches Leben im Zeitungswesen verbracht haben.

30 Pauli an Aniella Jaffé, 1. April 1953 (PWB IV/2, 104).

31 Pauli an Aniella Jaffé, ca. 10./11. April 1953 (PWB IV/2, 109). 
Wolfgang Paulis Verhältnis zur Politik unterscheidet sich ebenfalls grundlegend von dem seiner Vorfahren. Grossvater Schütz hat zwar keine direkte politische Laufbahn eingeschlagen, sein Schrifttum lässt jedoch klare politische Positionen erkennen. Ebenso, wenn nicht noch radikaler, war es bei dessen Tochter Bertha Pauli, der Mutter des Physikers. Verglichen mit diesen beiden war Wolfgang überhaupt kein homo politicus, zumindest nicht was das Bedürfnis betraf, seine Einstellung zu politischen Sachfragen einer breiteren Öffentlichkeit kundzutun. In seinen Briefen lernen wir Pauli als einen Menschen kennen, der sich durchaus Gedanken über aktuelle politische Ereignisse machte, etwa im Fall der Affäre Oppenheimer. Seine Meinungsäusserung zu diesen Themen beschränkte sich jedoch stets auf seinen unmittelbaren Bekanntenkreis. Insbesondere nach dem Ende des Zweiten Weltkriegs, dessen Schlusspunkt der Einsatz der ersten Atomwaffen bildete, schien das Bedürfnis nach öffentlicher politischer Artikulation bei den Physikern vieler Staaten besonders ausgeprägt zu sein. Berühmte Kollegen wie Heisenberg und Bohr nahmen Stellung zum Problem des Rüstungswettlaufs und anderen Themen wie der zunehmenden Konfrontation zwischen Ost und West. Pauli war demgegenüber der Ansicht, dass die Wissenschaft keinen allzu grossen Einfluss auf die politischen Ereignisse einer Epoche haben könne. Während die «Politik treibenden Professoren $»^{32}$ stets das Schicksal der gesamten Menschheit im Auge hatten, galt Paulis Aufmerksamkeit den individuellen Schicksalen, die seinem Denken weitaus zugänglicher waren.

Everything which is 'large scale' is too inhuman to be accessible to my sentiment, so I prefer to think about individual destinies, to stay human and to look how these individual fates are reflecting also a larger collective situation ${ }^{33}$.

In merkwürdigem Gegensatz zu diesen Aussagen steht eine Bemerkung seines ehemaligen Klassenkameraden Erich Hula über Wolfgang Pauli, die 1968, ein halbes Jahrhundert nach deren Matura, zu Papier gebracht wurde. Darin heisst es:

Nach Ausbruch des Ersten Weltkrieges erwachte in ihm ein leidenschaftliches Interesse für Politik, das sicherlich auch von seiner sozialistisch orientierten und schriftstellerisch tätigen Mutter genährt wurde. Je länger der Krieg dauerte, desto schärfer wurde seine Opposition gegen ihn und überhaupt gegen das ganze $<$ Establishment ${ }^{34}$.

Paulis früher Briefwechsel aus den zwanziger Jahren scheint die Aussage seines Mitschülers nicht zu bestätigen. Von Politik ist in diesen schriftlichen Zeugnissen nicht die Rede. Vielmehr scheint sich in dem jungen Physiker

32 Pauli an R. Schafroth, 17. Nov. 1953 (PWB IV/2, 358).

33 Pauli an N. Bohr, 6. Juni 1950 (PWB IV/1,111).

34 Zitiert nach K. v. Meyenn, «Wolfgang Pauli (1900-1958): Antimetaphysisch statt katholisch getauft», in: Hundert Jahre Gymnasium Gymnasiumstrasse (Wien 1985) 47. 
ein drastischer Sinneswandel vollzogen zu haben. Dafür spricht auch eine Bemerkung Paulis aus dem Jahr 1955, in der er seine Eindrücke am Beginn der zwanziger Jahre so umschreibt: «Der Krieg war aus, bei Sommerfeld war ich in das richtige Fahrwasser gekommen, was kümmerten mich als jungen Menschen dann noch die politischen und wirtschaftlichen Verhältnisse in Deutschland und Österreich?» ${ }^{35}$ Möglicherweise war es in jenen Tagen zu einer weitgehenden Reduzierung seiner politischen Interessen gekommen zugunsten einer um so intensiveren Konzentration auf seine wissenschaftlichen Aktivitäten.

Interessant ist vor diesem Hintergrund Paulis Einstellung zu den pazifistischen Strömungen, die insbesondere in den 50er Jahren in Wissenschaftlerkreisen sehr verbreitet waren. Erinnern wir uns daran, dass seine Mutter ebenso wie seine Schwester sehr stark vom Pazifismus geprägt waren. Auch der Physiker war ein durchaus friedliebender Mensch. Er vermied es jedoch, öffentliche Aufrufe zur weltweiten Abrüstung wie zum Beispiel die Initiative von B. Russell aus dem Jahr 1955 zu unterzeichnen.

Eine signifikante Abweichung Wolfgang Paulis von seinem unmittelbaren Wiener Umfeld bildet auch sein Verhältnis zu religiösen Fragen. Während der junge Physiker offenbar keinerlei Interesse an Diskussionen zu dieser Thematik besass, ja schliesslich sogar der katholischen Kirche den Rücken kehrte, zeigte sich der spätere Pauli durchaus an religiösen Erörterungen interessiert. Derartige Überlegungen, die er beispielsweise mit Niels Bohr diskutierte, zeigen ihn uns als einen Denker mit einer grossen Affinität zum Mystizismus, der darüber hinaus sehr stark von der östlichen Gedankenwelt etwa eines Lao-tse beeinflusst war. Mit der Vorstellung eines menschenähnlichen Gottes, wie sie sowohl dem Christentum als auch dem Judentum eigen ist, konnte sich Pauli nicht anfreunden. Seine anfängliche Abstinenz in religiösen Dingen dürfte wohl nicht zuletzt auf seinen überragenden Mentor Ernst Mach zurückzuführen sein, der aus seinem Antiklerikalismus keinen Hehl machte. Demgegenüber standen sowohl Paulis Grossvater Friedrich Schütz als auch seine Mutter durchaus auf dem Boden der christlich-europäischen Tradition. Dies war keineswegs im Widerspruch zu ihrer mehrfach geäusserten Kritik am Klerikalismus des damaligen Österreich, der vor allem wegen seiner politischen Ambitionen abgelehnt wurde. Aus den Schriften von Friedrich Schütz geht hervor, dass seine Einstellung zu religiösen Fragen keineswegs einem Atheismus gleichgesetzt werden kann. Vielmehr zeigte er sich einem «wahren Christentum» durchaus nicht abgeneigt. An einigen Stellen wird sogar eine gewisse Sympathie mit dem Protestantismus

35 Zitiert nach $P W B$ I, 33.

236 
erkennbar. Möglicherweise ist vor diesem Hintergrund Bertha Paulis Wechsel von der katholischen zur evangelischen Kirche zu sehen.

Von besonderem Interesse ist für uns die Wechselwirkung zwischen Vater und Sohn, zumal sie für die Ausbildung und Förderung der naturwissenschaftlichen Neigungen des späteren Physikers von ausschlaggebender Bedeutung gewesen sein dürfte. Wie erwähnt, zeigte der Vater Wolfgang Pauli sen. bereits während seiner Studienzeit an der Prager Universität ein ausgeprägtes Interesse an medizinischer Grundlagenforschung, wobei vor allem physikalische Aspekte eine gewichtige Rolle spielten. Ebenfalls als bedeutsam einzuschätzen ist die Bekanntschaft des Vaters mit Ernst Mach. Von den Prager Studententagen bis zu Machs Tod blieb Wolfgang Pauli sen. seinem Mentor in aufrichtiger Verehrung verbunden. Als besondere Auszeichnung darf der Umstand gelten, dass Ernst Mach die Patenschaft für den Sohn seines ehemaligen Schülers übernahm. Mach schenkte seinem Patenkind einige seiner von ihm selbst verfassten Bücher und gab dem Vater Ratschläge für weiterführende Literatur. Es darf als sicher angenommen werden, dass die Grundzüge der Machschen Philosophie einen nicht unerheblichen Einfluss auf den heranwachsenden Jüngling hinterlassen haben. Der Vater scheint diese geistige Patenschaft durchaus aktiv unterstützt zu haben. Dies lässt sich beispielsweise erkennen, wenn er in einem Brief an Mach nicht ohne Stolz vermerkt, dass sein Sohn dessen Mechanik «sehr fleissig» benützt. Auch die Empfehlung Machs, «einen älteren erfahreneren Mathematiker zu Rate zu ziehen» scheint bei Wolfgang Pauli sen., der seinen Sohn für ein mathematisches Genie hielt, auf fruchtbaren Boden gefallen zu sein. So nahm der Gymnasiast Privatunterricht bei dem Physiker Hans Bauer, der damals im höheren Schuldienst tätig war. 